\title{
Hepatocarcinoma y trombosis portal diagnosticados por ecografía y tomografía computada en pacientes cirróticos chilenos en un hospital público, 2004 - 2011
}

Drs. Pamela Sierra $S^{(1)}$, Daniela Pivcevic $C^{(1)}$, Andrés Retamal $C^{(1)}$, Patricio Latorre $B^{(1)}$, Jorge Contreras $B^{(2)}$, Claudio Silva $F^{(3)}$.

1. Becado de Radiología, Facultad de Medicina Clínica Alemana - Universidad del Desarrollo. Santiago, Chile.

2. Gastroenterólogo, Clínica Alemana de Santiago. Santiago, Chile.

3. Radiólogo, Clínica Alemana de Santiago. Facultad de Medicina Clínica Alemana - Universidad del Desarrollo. Santiago, Chile.

Hepatocellular carcinoma and portal vein thrombosis diagnosed by sonography and ct scan in chilean cirrhotic patients between 2004 and 2011

\begin{abstract}
There is no available data on prevalence of hepatocellular carcinoma (HCC) and portal thrombosis $(P T)$ in Chilean cirrhotic patients. Objectives: To evaluate the development of these entities and the role ultrasound (US) may play as the imaging method of choice in their diagnostic approach. Material and methods: $A$ cohort study of patients with diagnosis of cirrhosis by US or CT scans between 2004 and 2008. We evaluated both demographic and clinical records, along with disease development until 2011, by performing a retrospective review of their imaging findings. We investigated whether patients presented HCC and / or PT as detected by US / CT studies. Results: Two hundred and eleven (211) patients with an average age of 62.6 years were included. HCC was diagnosed in $10.4 \%$ of cases, whilst PT was observed in $4.3 \%$ of patients. A $33 \%$ of PT occurred in association with HCC and exhibited worse survival rates. Ultrasonographic studies yielded suspicious results in a significant percentage of cases, thus CT scans were performed to confirm the diagnosis. Conclusions: Ultrasound examination appears to be a useful tool for detecting complications of cirrhosis.

Keywords: Alcoholism, Cirrhosis, Hepatitis C Virus, Hepatocellular carcinoma, Portal vein thrombosis.
\end{abstract}

Resumen: No existen datos en pacientes cirróticos chilenos de prevalencia de hepatocarcinoma(CHC) y trombosis portal(TP).

Objetivos: Evaluar el desarrollo de estas complicaciones y el rol que podría jugar el ultrasonido (US) como primer método de imagen en su aproximación diagnóstica. Material y métodos: Estudio de cohorte en pacientes diagnosticados mediante US o TC de cirrosis entre 2004 y 2008. Se evaluaron datos demográficos, clínicos, y su evolución hasta 2011, realizando revisión retrospectiva de sus estudios imagenológicos. Se investigó si presentaron HCC y/o TP que fueran detectados mediante US/TC.

Resultados: Se incluyeron 211 pacientes, con un promedio de 62,6 años. En el 10,4\% se diagnosticó HCC y en el 4,3\% TP. El 33\% de TP ocurrieron asociadas a CHC y determinaron peor sobrevida. EI US fue sospechoso en un porcentaje significativo de los casos, motivando la realización de TC confirmatoria. Conclusiones: EI US aparece como una herramienta útil para la detección de complicaciones de cirrosis.

Palabras clave: Alcoholismo, Cirrosis, Hepatocarcinoma, Trombosis Portal, Virus Hepatitis C.

Sierra $\boldsymbol{P}$ y cols. Hepatocarcinoma y trombosis portal diagnosticados por ecografía y tomografía computada en pacientes cirróticos chilenos en un hospital público, 2004 - 2011. Rev Chil Radiol 2012; 18(1): 18-21.

Correspondencia: Dra. Pamela Sierra / pamess@gmail.com

Trabajo recibido el de 24 noviembre de 2011, aceptado para publicación el 09 de marzo de 2012.

\section{Introducción}

La cirrosis determina un importante problema de salud en el mundo, y forma parte de las 10 primeras causas de muerte ${ }^{(1)}$. Está definida por la Organización Mundial de la Salud como un proceso de afectación del parénquima hepático en forma difusa, caracterizado por fibrosis y conversión de la arquitectura hepática normal en nódulos ${ }^{(2)}$. Las principales causas de cirrosis descritas en el mundo son la infección crónica por virus hepatitis $\mathrm{C}(\mathrm{VHC})$, virus hepatitis $\mathrm{B}(\mathrm{VHB})$ y el consumo de alcohol(3-6). El diagnóstico de cirrosis por ultrasonido está ampliamente descrito en la literatura ${ }^{(2,7)}$.

Algunas de las principales complicaciones de la cirrosis son el carcinoma hepatocelular $(\mathrm{CHC})$ y la trombosis portal (TP) ${ }^{(8)}$. La cirrosis da cuenta del 80 $90 \%$ de los casos de $\mathrm{CHC}$ en el mundo(3). La cirrosis por VHB y VHC da cuenta del 50 y $25 \%$ de los $\mathrm{CHC}$ en el mundo respectivamente ${ }^{(5)}$ y están descritas como su principal causa(6). La incidencia de $\mathrm{CHC}$ en cirróticos es de $1-4 \%$ por año, aunque esta cifra varía 
según su causa, siendo más frecuente en hombres que mujeres y en mayores de 65 años ${ }^{(6)}$.

$\mathrm{El} \mathrm{CHC}$ en etapas tempranas es clínicamente silente y cuando es detectado porque se vuelve sintomático su sobrevida a 5 años es $<$ al $5 \%{ }^{(9)}$. Cuando se diagnostica en etapa inicial su sobrevida es $>50 \%$ a 5 años para aquellos que se someten a la terapia estándar actual (Resección + Trasplante Hepático). Dada la mejoría en la capacidad de tratar los $\mathrm{CHC}$ está la importancia de detectarlos en fases precoces, en los pacientes de mayor riesgo ${ }^{(10)}$.

El riesgo de desarrollar TP en cirrosis se ha relacionado a la severidad de la enfermedad hepática y a la presencia de trastornos protrombóticos hereditarios. Se ha estimado que la TP se presenta aproximadamente en el $11 \%$ de los cirróticos ${ }^{(11,12)}$, y cuando se diagnostica TP en el contexto de un paciente cirrótico debe descartarse siempre la coexistencia con $\mathrm{CHC}^{(13)}$.

El diagnóstico de $\mathrm{CHC}$ se basa en imágenes o biopsia $^{(10)}$. El diagnóstico radiológico de $\mathrm{CHC}$ no requiere biopsia si los hallazgos característicos están presentes cuando el nódulo mide $2 \mathrm{~cm}$ o más. Requiere sólo un estudio contrastado trifásico, ya sea tomografía computada $(\mathrm{TC})$ o resonancia magnética $(\mathrm{RM})^{(14)}$. No se ha determinado en la actualidad un protocolo o programa de seguimiento costo efectivo que se aplique universalmente a los pacientes cirróticos. Las últimas guías han recomendado la vigilancia basada en US y el intervalo recomendado de screening es 6 meses $^{(10)}$.

En Chile la cirrosis hepática es una enfermedad de alta prevalencia (aunque su cifra exacta no ha sido estudiada) y se han descrito como causas principales el consumo de alcohol y la hepatitis crónica por virus de la hepatitis $\mathrm{C}(\mathrm{VHC})^{(13)}$. Sin embargo, no existen datos que describan la frecuencia de aparición de complicaciones como CHC y TP, así como tampoco se ha logrado protocolizar, estandarizar y aplicar el seguimiento de estos pacientes dado el difícil acceso a métodos diagnósticos como son el ultrasonido (US), TC y RM.

De acuerdo a lo expuesto, el principal propósito del presente estudio fue presentar la frecuencia de estas complicaciones en una muestra de pacientes cirróticos del sistema público e identificar el rol que juega el US como primera modalidad de imagen en su diagnóstico.

\section{Materiales y métodos}

Estudio de cohorte retrospectivo, en que se incluye a todos los pacientes con cirrosis diagnosticados por imágenes, ya sea mediante US o TC, entre enero de 2004 y junio de 2008 en el Servicio de Radiología del Hospital Padre Hurtado de Santiago.

Los pacientes incluidos en este estudio pertenecen a una población de bajos recursos, por lo que sus posibilidades de acceso a técnicas diagnósticas y terapéuticas de sus distintas patologías se limitan sólo a este centro.

Se evaluaron datos demográficos (edad y sexo), clínicos (etiología de la cirrosis) y su evolución natural hasta julio de 2011, realizando una revisión retrospectiva de los informes imaginológicos de estos pacientes y se investigó si presentaron $\mathrm{CHC}$ y/o TP que fueran detectados mediante US o TC. En el subgrupo de pacientes con $\mathrm{CHC}$ se investigó su sobrevida. No se dispuso de RM en nuestro servicio.

\section{Criterios de exclusión}

- Pacientes con diagnóstico clínico de daño hepático crónico entre enero 2004 y junio 2008, pero sin método de imagen diagnóstica confirmatoria en este intervalo de tiempo.

- Pacientes con ultrasonido sospechoso para $\mathrm{CHC}$, pero que no se realizaron TC confirmatoria.

- Pacientes con lesiones focales hepáticas detectadas en TC sólo con fases sin contraste y/o venosa.

- Se excluyó del diagnóstico de $\mathrm{CHC}$ a lesiones que no cumplieran con los criterios establecidos por la Asociación Americana para Estudio de Enfermedades Hepáticas (AASLD) ${ }^{(10)}$.

\section{Evaluación ultrasonográfica}

Un radiólogo experimentado realizó ecotomografía abdominal evaluando dirigídamente el parénquima hepático, utilizando ecógrafos ATL 5000 (año 2004) y TOSHIBA I'Style 3800 (2008), con transductor convexo de 2-5 MHz. Evaluando el tamaño (normal/disminuido/ aumentado), homogeneidad del parénquima (homogéneo/heterogéneo), los contornos hepáticos (lisos/ nodulares o irregulares) y la presencia de lesiones focales (hipo/hipervasculares), hallazgos que se consignan en el informe radiológico.

En aquellos casos requeridos por la indicación médica se realizó estudio Doppler color dirigido a las venas porta, mesentérica superior y supra hepáticas, no estando protocolizado como parte del estudio rutinario abdominal de los pacientes cirróticos.

\section{Evaluación por tomografía computada}

Un radiólogo experimentado analizó tomografías computadas de abdomen trifásicas, utilizando un tomógrafo computado multicorte de 16 canales (Toshiba Aquilion), evaluando dirigídamente el parénquima hepático; sus contornos (lisos/nodulares o irregulares); tamaño (normal, disminuido, aumentado); homogeneidad del parénquima (homogéneo/heterogéneo); permeabilidad de la vena porta (permeable/trombosada); la presencia de lesiones focales y su cantidad (únicas/múltiples), su tamaño mayor y su comportamiento en las distintas fases del estudio (no contrastado/arterial/venoso), hallazgos que se consignan en el informe radiológico.

\section{Resultados}

Se ingresa al estudio a los pacientes diagnosticados de cirrosis entre los años 2004 y 2008. De los pacientes estudiados, un total de 211 (108 hombres y 103 mujeres) cumplieron con los criterios de inclusión establecidos. Estos tenían un promedio de 62,6 años de edad (rango entre 30 y 88 años). 
En el $41,2 \%$ de los casos (87 pacientes) se determinó la etiología de la cirrosis (Gráfico 1). De éstos, la causa más frecuente fue el consumo crónico de alcohol $(57,5 \%)$ seguido en orden de frecuencia por infección por VHC $(9,2 \%)$ y hepatitis autoinmune $(6,9 \%)$.

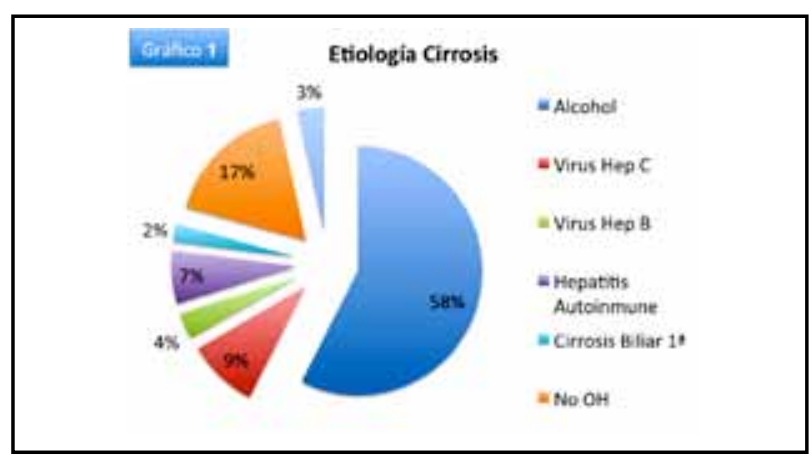

El 10,4\% de los pacientes (11 mujeres y 11 hombres) desarrollaron $\mathrm{CHC}$ entre el momento del diagnóstico y julio 2011. De éstos, la sobrevida actual es del 9,1\% (2 pacientes).

Las etiologías más comunes de la cirrosis en los pacientes con $\mathrm{CHC}$ fueron en orden decreciente: Consumo crónico de alcohol, infección por VHC e infección por VHB.

El $4,3 \%$ de los pacientes ( 6 hombres y 3 mujeres) desarrollaron TP entre el momento diagnóstico de cirrosis y julio 2011. De éstos, el 33,3\% (3 casos) coexistieron con $\mathrm{CHC}$. Los restantes casos se desarrollaron en distintos contextos clínicos tales como: Peritonitis bacteriana espontánea (PBE), Pielonefritis aguda, Diabetes Mellitus tipo II descompensada, entre otras.

Los pacientes diagnosticados de $\mathrm{CHC}$ con TC multicorte trifásica se realizaron ultrasonido previo en el $63,3 \%$ de los casos (14 pacientes), y este método sugirió realización de TC confirmatoria por sospecha de lesión focal de aspecto neoplásico en el 92\% de los casos (13 de 14 ultrasonidos).

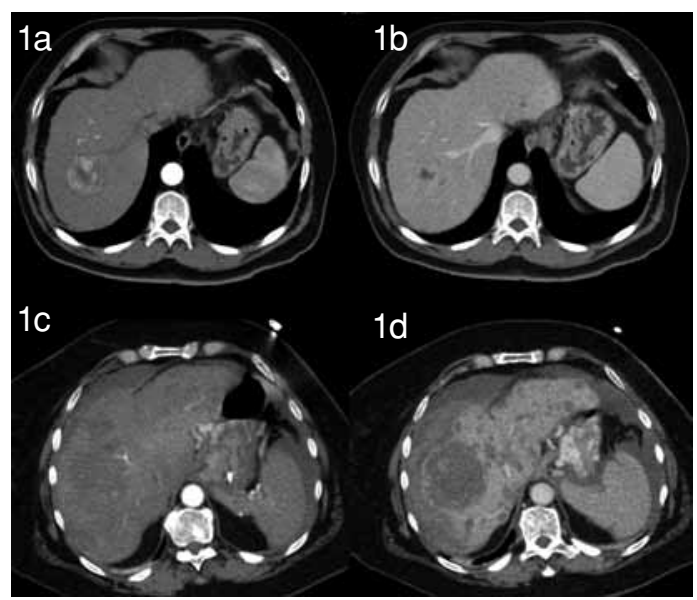

Figura 1. Tomografía computada trifásica en fase arterial y portovenosa que presenta lesiones compatibles con hepatocarcinoma unifocal ( $A$ y B) y multifocal (C y D).
Los pacientes diagnosticados de TP se realizaron ultrasonido previo en el $33,3 \%$ de los casos (3 pacientes), y este método sugirió la realización de TC confirmatoria en el $100 \%$ de los casos. En los restantes pacientes se realizó TC como primer método de imagen y diagnóstico de la TP.

El análisis de los datos demostró que la mediana de sobrevida de los $\mathrm{CHC}$ identificados por imágenes es de 22 días (Curva 1), y el test de log-rank para TP es altamente significativo $(p<0,001)$, reflejando que TP es un factor significativo para predecir mortalidad. Es más, en la curva Kaplan-Meier para trombosis portal se observa que cae en vertical con una mediana de sobrevida en su presencia de cinco días (Curva 2).

No hubo diferencias estadísticamente significativas respecto de la sobrevida de los pacientes si el $\mathrm{CHC}$ era solitario o multicéntrico.
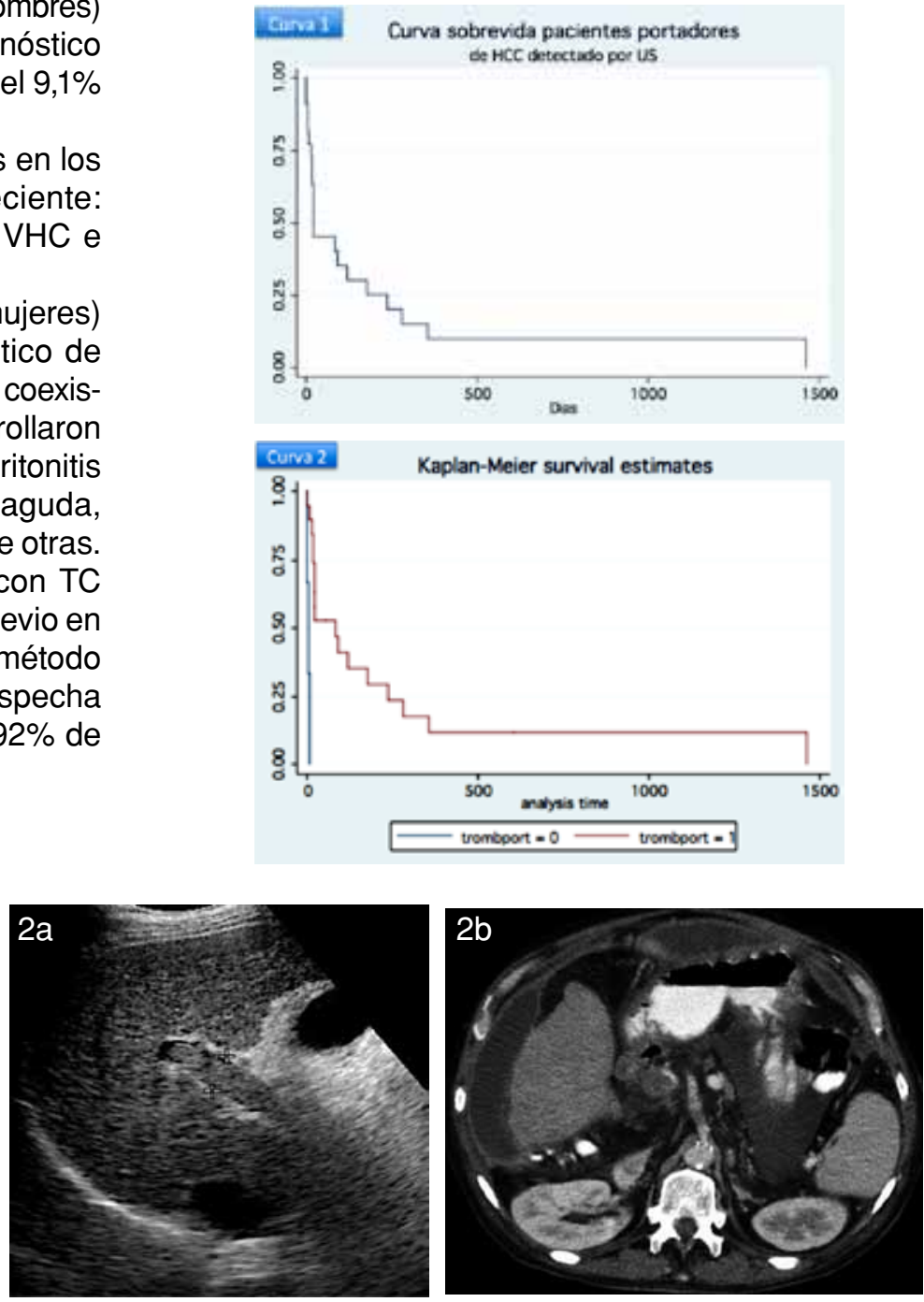

Figura 2a. Ocupación por material hipoecogénico en vena porta, la cual no presenta flujo a la evaluación con Doppler color y pulsado, altamente sugerente de trombosis portal.
Figura 2b. Tomografía computada en fase portovenosa, que confirma trombosis portal al identificarse material hipodenso en vena porta. 


\section{Discusión}

La cirrosis es una enfermedad de alta prevalencia en nuestro país, determina significativa morbilidad ${ }^{(1)}$ por sus distintas complicaciones (CHC, TP, PBE, hemorragia digestiva, entre otras) y alta frecuencia de hospitalizaciones, y un pronóstico de sobrevida a largo plazo muy pobre. Situación que justifica determinar con mayor exactitud su frecuencia, sus complicaciones y el pronóstico de los pacientes portadores de esta enfermedad de nuestro sistema público de salud.

Al ser retrospectivo nuestro estudio, se analizaron resultados y hasta esta fecha no estaba protocolizado el estudio de estos pacientes (tal como el hecho de no estudiar dirigídamente el sistema venoso con Doppler). Sin embargo, los resultados demuestran que el US es una técnica altamente eficaz para la detección de $\mathrm{CHC}$ en esta serie de casos con daño hepático crónico (DHC) sintomático.

El diagnóstico imagenológico de estas complicaciones demostró ser tardío (en el 91\% de los casos), lo que se ve reflejado en la corta sobrevida de la muestra.

Estos resultados apoyan la necesidad del uso de screening US para evitar la consulta de los pacientes con $\mathrm{CHC}$ en etapa avanzada, no candidatos a terapia.

El uso de la TC trifásica como método de confirmación diagnóstica de $\mathrm{CHC}$ es indiscutido, pero en el sistema público es un recurso escaso, por lo que proponemos potenciar la capacidad del sistema público en el uso del US como método de seguimiento en el DHC, dado su fácil acceso y bajo costo, optimizando el recurso de la TC. Esta medida permitiría derivar prontamente a este estudio confirmatorio sólo el grupo selecto de estos pacientes en que se sospecha que las presenten, para que puedan acceder a un tratamiento oportuno.

El principal aporte de este estudio es la utilización del US como herramienta para la detección de estas complicaciones en los pacientes cirróticos, aprovechando y potenciando en el sistema público de salud este método diagnóstico de fácil acceso, sin el riesgo de efectos adversos conocidos por el uso de medio de contraste y la radiación, para que se realice rutinariamente en el control habitual de estos pacientes.

Dados nuestros resultados proponemos aplicar rigurosamente el protocolo de seguimiento actualmente recomendado por la AASLD ${ }^{(10)}$, para así poder ofrecer mejores expectativas, tanto en calidad como en pronóstico de sobrevida a largo plazo, dado que el diagnóstico de $\mathrm{CHC}$ en un paciente cirrótico lo traslada como prioridad a la lista de trasplante y. por lo tanto, a la posibilidad de curar su enfermedad ${ }^{(10)}$.

\section{Conclusiones}

El consumo de alcohol es la principal causa de cirrosis en nuestra población y es además la causa más frecuente de $\mathrm{CHC}$ en pacientes cirróticos chilenos, seguido por la infección por VHC.

La incidencia de $\mathrm{CHC}$ en nuestra serie fue del $10,4 \%$, confirmando lo descrito en series extranjeras.

La incidencia de TP fue del 4,3\%, cifra menor a la descrita en series extranjeras y chilenas, y su coexistencia con $\mathrm{CHC}$ no fue el factor más frecuente en globo, aunque sí como factor aislado.

Los resultados revelan que, ante ausencia de seguimiento US la consulta de los pacientes de alto riesgo de $\mathrm{CHC}$ es tardía, lo que conlleva una baja sobrevida.

\section{Bibliografía}

1. Alonso F, Garmendia M, De Aguirre M, Searle J. Análisis de la tendencia de la mortalidad por cirrosis hepática en Chile: Años 1990 a 2007. Rev Med Chile 2010; 138: 1253-1258.

2. Wilson S, Withers C. El Hígado. Rumack C, Wilson S, Charboneau J. Diagnóstico por ecografía. 3aㅡ Ed. Génova: Elsevier; 2006. pp. 77-145.

3. Bartolomeo N, Trerotoli P, Serio G. Progression of liver cirrhosis to $\mathrm{CHC}$ : an application of hidden Markov model. BMC Medical Research Methodology 2011; 11: 38.

4. Carrilho F, Kikuchi L, Branco F, Sandoval C, Aves A. Clinical and epidemiological aspects of hepatocellular carcinoma in Brazil. Clinics 2010; 65(12): 1285-1290.

5. Jang J, Chung R. Chronic Hepatitis C. Gut and Liver 2011; (2): 117-132.

6. Sanyal A, Kew Yoon S, Lencioni R. The Etiology of Hepatocellular Carcinoma and Consequences for Treatment. The Oncologist 2010; 15(suppl 4): 14-22.

7. Di Lelio A, Cestani C, Lomazzi A, Beretta L. Cirrhosis: Diagnosis with Sonographic Study of the Liver Surface. Radiology 1989; 172: 389-392.

8. Chung R, Podolsky D. Cirrosis y sus complicaciones. Kasper D, Braunwald E, Fauci A, Hauser S, Longo D, Jameson J. Harrison Principios de Medicina Interna. 16 Ed. México D.F.: Mc Graw-Hill; 2005; pp. 2046-2058.

9. Clark H, Forrest W, Kavanagh P, Ho C, Shen P, Zagoria R. Staging and Current Treatment of Hepatocellular Carcinoma. RadioGraphics 2005; (25): S3-S23.

10. Bruix J, Sherman M. AASLD Practice guideline Management of Hepatocellular Carcinoma: An Update. Hepatology 2011; 53(3): 1020.

11. Amitrano L, Guardascione M, Brancaccio V, Margaglione M, Manguso F, lannaccone L, et. al. Risk factors and clinical presentation of portal vein thrombosis in patients with liver cirrosis. Journal of Hepatology 2004 (40): 736-741.

12. Naranjo J, Contreras J, Miranda J, Naranjo B, Pacheco $\mathrm{S}$, Retamales B, Melys A, et. al. Incidencia y factores pronósticos de trombosis portal en cirrosis hepática. Gastroenterol. Latinoam 2006; 17: 452.

13. Zapata R, Pérez-Ayuso R, Contreras J, Fuster F, Márquez S, Becerra M. Estudio etiológico multicéntrico de la cirrosis hepática en Chile. Gastroentero latinoam 2008; 19(4): 305.

14. Alústiza J, Martí-Bonmatí. Radiología de las enfermedades hepáticas: enfermedad hepática difusa y lesión focal hepática. Del Cura J, Pedraza S, Gayete A. Radiología Esencial. 1ª Ed. Madrid: Editorial Médica Panamericana; 2010; pp. 512-527. 